\title{
Diversity Multiplexing Tradeoff in MIMO Frequency Selective Channels with Partial CSIT
}

\author{
Yong Peng, Kaushik Josiam, and Dinesh Rajan, Senior Member, IEEE
}

\begin{abstract}
In this letter, we derive the optimal diversitymultiplexing tradeoff for a frequency selective MIMO channel with resolution-constrained channel feedback. The additional degree of freedom provided by the channel multipaths is succinctly captured in the tradeoff characterization. For instance, in a $L$-path SISO channel, with $K$-level feedback, the maximum diversity increase is exponential in $K$.
\end{abstract}

Index Terms - Wideband, MIMO, power control, diversity.

\section{INTRODUCTION}

$\mathbf{T}$ HE tradeoff between transmission data rate and error rate in a multiple antenna system is elegantly characterized by the diversity-multiplexing tradeoff (DMT) in [1]. Recently, the optimal DMT with power and rate control using partial channel state information at the transmitter (CSIT) in MIMO flat fading channels is derived in [2], [3].

Wideband channels are typically frequency selective and the transmit symbols experience multipath fading. The multiple channel paths provide an additional degree of freedom which can be exploited to improve both diversity gain (DG) and multiplexing gain (MG). This improvement has been characterized using the optimal DMT with no CSIT in [4], [5]. In this letter, we characterize the DMT of a MIMO wideband channel with CSIT. This CSIT is obtained via an instantaneous, error-free, bandwidth constrained feedback link. We evaluate the outage probability using the analysis in [6] to convert the frequency selective channel into a set of parallel fading channels. We first modify the wideband channel into an equivalent set of parallel fading channels using the approach in [6]. The optimal DMT with partial CSIT is then immediate when the feedback, power and rate control strategies derived in [2] are applied to the set of parallel fading channels. The feedback is a deterministic mapping of the channel to an integer index which is used for power and rate control. Since our analysis closely follows [2], we omit the proof for the main results and only indicate relevant adaptations to the wideband channel. The DMT with partial CSIT reveals an important insight: In a SISO wideband channel with $L$ multipaths, the $K$-index feedback increases the maximum diversity by $\sum_{k=1}^{K} L^{k}$, which is an exponential increase in $K$. In contrast is a SISO flat fading channel, the maximum diversity only increases linearly with $K$ [2].

\section{SYSTEM MODEL}

Consider a MIMO wideband block fading channel with $N_{t}$ transmit and $N_{r}$ receive antennas. The wideband channel

Manuscript received February 13, 2008. The associate editor coordinating the review of this letter and approving it for publication was A. Haimovich.

The authors are with the Wireless Networking Lab, Dept. of Electrical Engineering, Southern Methodist University, Dallas, TX 75275, USA (\{ypeng, kjosiam, rajand\}@engr.smu.edu).

Digital Object Identifier 10.1109/LCOMM.2008.080228. between the $j^{\text {th }}$ transmit and the $i^{\text {th }}$ receive antenna is a vector $\hat{\mathbf{h}}_{i j}^{q}=\left[\hat{h}_{i j}^{q}(0), \ldots, \hat{h}_{i j}^{q}(L-1)\right]$ composed of $L$ distinct multipath components that are i.i.d. $\sim \mathbb{C N}(0,1)$. Let there be $T$ channel uses per fading block and $\eta=\min \left\{N_{t}, N_{r}\right\}$.

Computing the channel mutual information is a key step in deriving the DMT. Numerous approaches [7]-[9] have been proposed to compute the mutual information for a wideband channel. A popular approach is to use orthogonal frequency division multiplexing (OFDM) to convert a wideband channel into a set of parallel flat fading channels and then compute the mutual information [6], [7]. We show in the following section that OFDM is a simple and intuitive tool to fully capture the additional DG provided by the $L$ multipaths.

In an OFDM transmitter, data are processed in blocks of length $N$ and transformed by inverse discrete Fourier transform (IDFT) to $N$ subcarriers. The transformed block of symbols is then lengthened to $N+L$ symbols by repeating the last $L$ symbols at the beginning of the block as a cyclic prefix (CP). At the receiver, the $\mathrm{CP}$ is removed, and the $N$ symbols are transformed back using a DFT. Such an approximation of the wideband channel by $N$ parallel channels is valid when $N \rightarrow \infty$ and $L \ll N$ [7]. The received signal, $\mathbf{Y}_{n}^{q}$, of size $N_{r} \times T$ at the $n^{t h}$ subcarrier in the $q^{t h}$ fading block is,

$$
\mathbf{Y}_{n}^{q}=\mathbf{H}_{n}^{q} \mathbf{X}_{n}^{q}+\mathbf{Z}_{n}^{q}, \quad n=0,1, \ldots, N-1
$$

where $\mathbf{X}_{n}^{q}$ is a $N_{t} \times T$ complex matrix of transmit symbols and $\mathbf{H}_{n}^{q}$ is the $N_{r} \times N_{t}$ channel matrix after DFT with elements, $h_{n}^{q}(i, j)=\sum_{l=0}^{L-1} \hat{h}_{i j}^{q}(l) e^{-\frac{j 2 \pi l n}{N}}$. Each element of the noise matrix $\mathbf{Z}_{n}^{q}$ is i.i.d. $\sim \mathbb{C N}(0,1)$. Superscript $q$ for block index is omitted in the sequel when it is clear from context.

The receiver is assumed to have perfect knowledge of the channels in each subcarrier. Define $\mathcal{H}=\left\{\mathbf{H}_{n}\right\}_{n=0}^{N-1}$. The feedback strategy which is similar to that in [2] is as follows: For each channel realization, the receiver sends the transmitter a noise free, instantaneous index $\mathcal{I}(\mathcal{H}) \in\{1, \ldots, K\}$, where positive integer $K$ is the feedback resolution. The feedback index is a deterministic mapping from a channel matrix to an integer index and corresponds to one of $K$ partitions of the space of channel matrices. The transmitter has $K$ transmit codebooks $\left\{\mathcal{C}_{i}=\left\{\mathbf{S}_{i}(1), \ldots, \mathbf{S}_{i}\left(M_{i}\right)\right\}\right\}_{i=1}^{K}$ each with rate $\left\{R_{i}\right\}_{i=1}^{K}$. Conditioned on the feedback index, $\mathcal{I}(\mathcal{H})=i$, the transmitter selects for transmission one of the codewords (each is of size $N_{t} \times T$ ) at random from the codebook, $\mathcal{C}_{i}$. The average total transmit power, $P_{i}$, when $\mathcal{I}(\mathcal{H})=i$ is given by $P_{i} \triangleq \frac{1}{T M_{i}} \sum_{u=1}^{M_{i}}\left\|\mathbf{S}_{i}(u)\right\|_{F}^{2}$ where $\|\cdot\|_{F}$ denotes the Frobenius norm of a matrix. Additionally, there is a long-term power constraint which is defined as $\lim _{Q \rightarrow \infty} \frac{1}{Q} \sum_{q=1}^{Q} \frac{1}{T}\left\|\mathbf{S}^{q}\right\|_{F}^{2} \stackrel{\text { a.s. }}{=} E_{\mathcal{H}}\left[P_{\mathcal{I}}\right] \leq$ SNR. The average data 
rate $R$ over infinite fading blocks is $R^{a . s .} \sum_{i=1}^{K} \operatorname{Pr}(\mathcal{I}(\mathcal{H})=$ i) $R_{i}$. Consequently, the average $\mathrm{MG}, r=\lim _{\mathrm{SNR} \rightarrow \infty} \frac{R}{\log \mathrm{SNR}}$.

The problem of finding optimal DMT with partial CSIT is closely linked to finding an optimal feedback design for wideband channels. Since we consider a slow fading channel, our objective is to compute the smallest outage error probability with a $K$-index feedback. In particular we are interested in the DG, $d_{o u t, K}^{*}(r)$ defined as the SNR exponent in the high SNR approximation of the outage probability, when the transmission rate is $r \log$ SNR. Note that throughout the letter, $\doteq$ indicates exponentially equality [1].

\section{OPTIMAL DMT FOR WIDEBAND TRANSMISSION WITH LIMITED FEEDBACK}

\section{A. Outage Analysis for Frequency Selective Channel}

Assume data symbols on all subcarriers are independent and the number of subcarriers $N=m L$, the mutual information for the MIMO-OFDM system [6] when $\mathcal{I}(\mathcal{H})=i$ is given by

$$
\begin{aligned}
& I\left(\mathbf{X}_{n} ; \mathbf{Y}_{n} \mid \mathcal{H}, \mathcal{I}(\mathcal{H})=i\right)=\sum_{n=0}^{N-1} \log \operatorname{det}\left(\mathbf{I}_{N_{r}}+P_{i} \mathbf{H}_{n} \mathbf{H}_{n}^{H}\right) \\
& =\sum_{l=0}^{L-1} \sum_{t=0}^{m-1} \log \operatorname{det}\left(\mathbf{I}_{N_{r}}+P_{i} \mathbf{H}_{l m+t} \mathbf{H}_{l m+t}^{H}\right) .
\end{aligned}
$$

In deriving (2), the $N$ subcarriers are split into $m$ groups, each of which has $L$ subcarriers over uniformly spaced indices. For large $m$, the subcarriers in a group are chosen from indices sufficiently far apart, that the fading on these subcarriers are uncorrelated and the gains due to frequency diversity can be ensured. Grouping subcarriers in this fashion when used in conjunction with a linear constellation precoder can extract maximum multipath diversity in OFDM systems [10]. Define $I\left(\mathcal{H}_{l}, \pi\right)=\sum_{l=0}^{L-1} \log \operatorname{det}\left(\mathbf{I}_{N_{r}}+\pi \mathbf{H}_{l} \mathbf{H}_{l}^{H}\right)$. Outage occurs when the mutual information in (2) falls below the desired instantaneous rate $R_{i}=m r_{i} \log$ SNR. Define the outage probability for this modified system as $\mathcal{O}\left(R_{i}, P_{i}\right)=\operatorname{Pr}\left[\sum_{t=0}^{m-1} I\left(\mathcal{H}_{l m+t}, P_{i}\right)<m r_{i} \log\right.$ SNR $]$. For any $x>0, \log (1+x)$ is a nonnegative function. Therefore, $\left\{\log \left(1+x_{t}\right)<1, \forall t=0, \ldots, m-1\right\} \subseteq\left\{\sum_{t=0}^{m-1} \log \left(1+x_{t}\right)<\right.$ $m\} \subseteq \bigcup_{t=0}^{m-1}\left\{\log \left(1+x_{t}\right)<1\right\}$. Hence, the outage can be bounded as $\operatorname{Pr}\left[I\left(\mathcal{H}_{l m+t}, P_{i}\right)<r_{i} \log \mathrm{SNR}\right] \leq P_{\text {out }, K} \leq$ $m \operatorname{Pr}\left[I\left(\mathcal{H}_{l m+t}, P_{i}\right)<r_{i} \log \mathrm{SNR}\right], 0 \leq t<m$. Notice that the upper and lower bounds of $P_{\text {out }, K}$ are defined by the same outage event. In the limit SNR $\rightarrow \infty$, the outage probability is,

$$
\mathcal{O}\left(R_{i}, P_{i}\right) \doteq \operatorname{Pr}\left[I\left(\mathcal{H}_{l m}, P_{i}\right)<r_{i} \log \mathrm{SNR}\right] .
$$

Thus, at high SNR, for any $N$, a set of appropriately chosen $L$ subcarriers are sufficient to capture the contribution of the wideband channel to the outage event.

\section{B. Optimal Tradeoff with Partial CSIT}

We focus on the DMT for single-rate transmission, i.e., all $K$ codebooks have the same rate $R$. The resolutionconstrained feedback is then utilized exclusively for transmit power control, where users set their transmit power $P_{i}$, depending on the feedback index $\mathcal{I}(\mathcal{H})=i$. Then, from (3), we see that the feedback $\mathcal{I}(\mathcal{H})$ and power codebook $\left\{P_{i}^{*}\right\}_{i=1}^{K}$ completely determine system outage. The objective is to find the optimum power codebook that minimizes system outage subject to an average power constraint, SNR. The optimum power codebook can be found as the solution to the constrained optimization problem characterized in Lemma 1, which is directly adapted from [2] for the wideband channel.

Lemma 1: For a given SNR and rate $R$, the outage minimizing power codebook $\left\{P_{i}^{*}\right\}_{i=1}^{K}$ solves the following optimization problem

$$
\begin{aligned}
& \left.\max _{0<P_{1}<\cdots<P_{K}} \quad P_{K} \quad \text { s.t. } \quad\left[\mathcal{O}\left(R, P_{K}\right)\right]+1-\mathcal{O}\left(R, P_{1}\right)\right] P_{1} \\
& +\sum_{i=2}^{K}\left[\mathcal{O}\left(R, P_{i-1}\right)-\mathcal{O}\left(R, P_{i}\right)\right] P_{i} \leq \text { SNR. }
\end{aligned}
$$

The optimal index mapping is given by

$$
\mathcal{I}^{*}(\mathcal{H})=\left\{\begin{array}{l}
1, \quad \text { if } I\left(\mathcal{H}_{l m}, P_{K}^{*}\right)<R \\
\min \left\{i: i \in\{1, \ldots, K\}, I\left(\mathcal{H}_{l m}, P_{i}^{*}\right) \geq R\right\} \quad \text { else }
\end{array}\right.
$$

Outage occurs only when even the maximum transmit power in the codebook, $P_{K}^{*}$, can not support the transmit rate, $R$. The transmit power for the best and worst channel conditions are mapped to the feedback index $\mathcal{I}^{*}(\mathcal{H})=1$, which suggests that the transmitter must save power when the channel is bad so that it may be used later [2]. Now, for any arbitrary power level, $\pi$, the outage probability, $\mathcal{O}(R, \pi)$, in the limit SNR $\rightarrow$ $\infty$ is a generalization of the result in [4] and is described in Lemma 2.

Lemma 2: For $r \in(0, L \eta)$, let $\pi$ be a function of SNR such that $\pi \doteq \operatorname{SNR}^{p}$ where $p$ is a finite constant and $p \geq 1$. Let $(x)^{+}=\max (x, 0)$. Then $\mathcal{O}(r \log \operatorname{SNR}, \pi) \doteq \operatorname{SNR}^{-D(r, p)}$, where

$$
\begin{gathered}
D(r, p) \triangleq \inf _{\alpha_{l i} \in \mathcal{A}^{\prime}} \sum_{l=0}^{L-1} \sum_{i=1}^{\eta}\left(2 i-1+\left|N_{t}-N_{r}\right|\right) \alpha_{l i} \\
\mathcal{A}^{\prime}=\left\{\alpha_{l i} \in \mathbb{R}^{L \eta} \mid \alpha_{l 1} \geq \cdots \geq \alpha_{l \eta} \geq 0\right. \\
\left.\forall l=0, \ldots, L-1, \sum_{l, i}\left(p-\alpha_{l i}\right)^{+}<r\right\} .
\end{gathered}
$$

The minimizing $\alpha^{*}$ can be explicitly computed using the Laplace principle given in [1], as

$$
\alpha_{l i}^{*}= \begin{cases}p & \forall l, i=1, \ldots, \beta-1, \\ p-r / L+p\lfloor r / L\rfloor & \forall l, i=\beta, \\ 0 & \forall l, i=\beta+1, \ldots, \eta .\end{cases}
$$

where $\beta=\eta-\lfloor r / p L\rfloor$.

The optimal DMT for the wideband channel is a solution to optimization problem (4). Using the index assignment in Lemma 1 and the outage probability computation in Lemma 2 , the outage probability for the wideband channel is described in the following theorem.

Theorem 1: For a single-rate MIMO wideband channel with $K$ quantization regions in the feedback, the outage upper bound is given recursively by

$$
d_{\text {out }, K}^{*}=D\left(r, 1+d_{\text {out }, K-1}^{*}(r)\right)
$$


where $d_{o u t, 0}^{*} \triangleq 0, \forall r$ and $D(r, p)$ is defined in (5).

The proof of Theorem 1 is similar to Theorem 1 in [2] and is therefore omitted. It is easy to show that the DMT is asymptotically achievable when $T \rightarrow \infty$ [1]. The achievability with finite $T$ is beyond the scope of this letter.

Theorem 1 extends directly to the case where the transmitter adapts both the power and rate. As shown in [2], the DMT with a $K$-index feedback is defined by a two rate codebook, the desired rate, $R=r \log$ SNR, and a minimum rate, $R_{\min }=$ $r_{\text {min }} \log$ SNR, along with $K$ power codebooks. The DMT for this adaptive rate transmission is,

$$
d_{\text {out }, K}^{*}\left(r, r_{\min }\right)=D\left(r_{\min }, 1+d_{\text {out }, K-1}^{*}\left(r, r_{\min }\right)\right)
$$

where $d_{o u t, 1}^{*}\left(r, r_{\text {min }}\right) \triangleq D(r, 1), \forall r \in\left[r_{\text {min }}, L \eta\right)$, and $r_{\text {min }} \in(0, L \eta)$.

\section{Special Cases}

1) No CSIT, i.e., $K=1$ : The optimal DMT is given by the piecewise linear function

$$
d_{\text {out }}^{*}(r)=D(r, 1)=L\left(N_{t}-r / L\right)\left(N_{r}-r / L\right) .
$$

In particular, $d_{\max }^{*}=L N_{t} N_{r}$ and $r_{\max }^{*}=L \eta$. Further for flat fading channels, i.e., $L=1$, the optimal DMT simplifies to $d_{\text {out }}^{*}=\left(N_{t}-r\right)\left(N_{r}-r\right)$ which concurs with [1].

2) Single antenna at one side, i.e., $\eta=1$ : The DMT is a straight line between $\left(0, \sum_{k=1}^{K}\left(L N_{t} N_{r}\right)^{k}\right)$ and $(L, 0)$, i.e.,

$$
\begin{aligned}
d_{\text {out }, K}^{*}(r) & =\left(1-\frac{r}{L}\right) \sum_{k=1}^{K}\left(L N_{t} N_{r}\right)^{k} \\
& =\left(1-\frac{r}{L}\right) \frac{L N_{t} N_{r}\left(\left(L N_{t} N_{r}\right)^{K}-1\right)}{L N_{t} N_{r}-1} .
\end{aligned}
$$

Note that for a SISO case $\left(N_{t}=N_{r}=1\right)$, with $L$ independent multipaths, the maximal DG grows as $\sum_{k=1}^{K} L^{k}$. Therefore, the value of the feedback is higher in a wideband system $(L \geq$ 2) than a flat fading system.

3) Diversity gain for extreme values of $r$ : For single-rate transmission, we have

$$
\lim _{r \rightarrow 0} d_{\text {out }, K}^{*}(r)=\sum_{k=1}^{K}\left(L N_{t} N_{r}\right)^{k} \text { and } \lim _{r \rightarrow r_{\text {max }}^{*}} d_{\text {out }, K}^{*}(r)=0 .
$$

For adaptive-rate transmission when $K \geq 2$, we have

$$
\lim _{r_{m i n} \rightarrow 0} d_{\text {out }, K}^{*}\left(r, r_{\text {min }}\right)=\left(L N_{t} N_{r}\right)^{K-1} D(r, 1)+\sum_{k=1}^{K-1}\left(L N_{t} N_{r}\right)^{k}
$$

where $D(r, 1)$ is given by (7). From (9), we can also derive $\lim _{r \rightarrow r_{\text {min }} \rightarrow 0} d_{\text {out }, K}^{*}\left(r, r_{\text {min }}\right)=\sum_{k=1}^{K}\left(L N_{t} N_{r}\right)^{k}$ and $\lim _{r \rightarrow r_{\text {max }}^{*} \rightarrow 0} d_{\text {out }, K}^{*}\left(r, r_{\text {min }}\right)=\sum_{k=1}^{K-1}\left(L N_{t} N_{r}\right)^{k}$. This result shows that with adaptive-rate transmission when $r_{\min }$ is small, even if we transmit at the maximum achievable rate in a SISO wideband channel, we can still achieve diversity which grows exponentially with $K$.

Fig. 1 shows the optimal DMT for single-rate and adaptiverate transmission $\left(r_{\min }=0.001\right)$ for different number of multipaths $L$ and feedback resolution $K$. For a given $K$, as $L$ increases, both the maximum rate and diversity increase. In

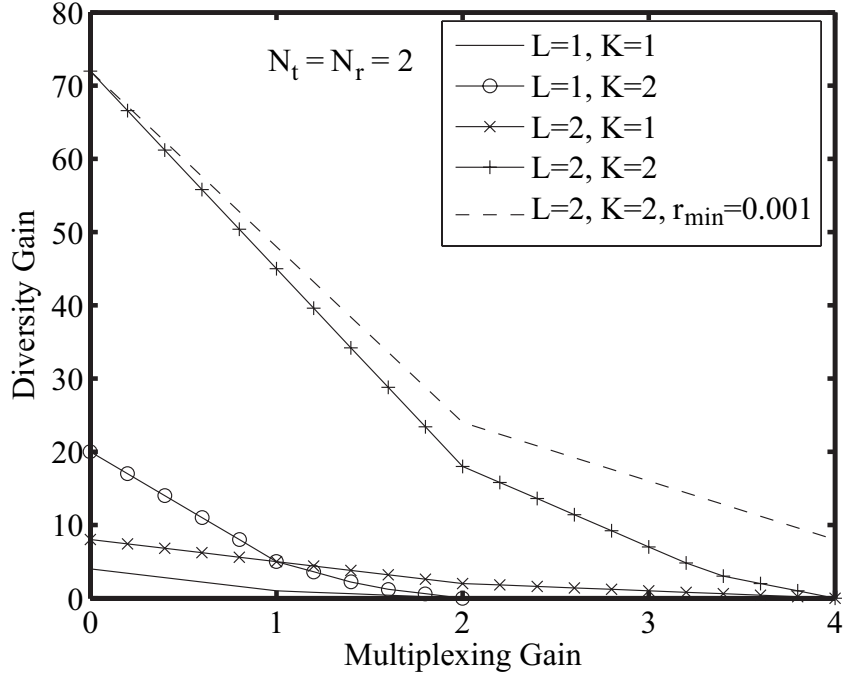

Fig. 1. Optimal DMT over a $2 \times 2$ channel.

contrast, for a fixed $L$, as $K$ increases, only the maximum diversity is increased. Also, the increase in maximum diversity as $K$ increases is greater for larger values of $L$. Further, using adaptive rate transmission allows nonzero diversity to be achieved even at the highest MG.

In this letter, the total power is split across all transmit antennas and subcarriers. Future work should investigate the effect of nonuniform power allocation on the D-M tradeoff.

\section{REFERENCES}

[1] L. Zheng and D. Tse, "Diversity and multiplexing: a fundamental tradeoff in multiple-antenna channels," IEEE Trans. Inform. Theory, vol. 49, no. 5, pp. 1073-1096, May 2003.

[2] T. Kim and M. Skoglund, "Diversity-multiplexing tradeoff in MIMO channels with partial CSIT," IEEE Trans. Inform. Theory, vol. 53, no. 8, pp. 2743-2759, Aug. 2007.

[3] H. Gamal et al., "The MIMO ARQ channel: diversity-multiplexingdelay tradeoff," IEEE Trans. Inform. Theory, vol. 52, no. 8, pp. 36013621, Aug. 2006.

[4] S. Yang et al., "Perfect space-time block codes for parallel MIMO channels," in Proc. ISIT, pp. 1949-1953, July 2006.

[5] A. Medles and D. Slock, "Optimal diversity vs multiplexing tradeoff for frequency selective MIMO channels," in Proc. ISIT, pp. 1813-1817, Sept. 2005.

[6] A. Scaglione, "Statistical analysis of the capacity of MIMO frequency selective Rayleigh fading channels with arbitrary number of inputs and outputs," in Proc. ISIT, p. 278, July 2002.

[7] D. Tse and P. Viswanath, Fundamentals of Wireless Communication. Cambridge Univ. Press, 2005.

[8] Z. Fan and L. Sharf, "The approximation of outage probability and the trade-off between capacity and diversity for the frequency-selective channel," in Proc. ISIT, pp. 1159-1163, July 2006.

[9] I. Telatar and D. Tse, "Capacity and mutual information of wideband multipath fading channels," IEEE Trans. Inform. Theory, vol. 46, no. 4, pp. 1384-1400, July 2000.

[10] Z. Liu et al., "Linear constellation precoding for OFDM with maximum multipath diversity and coding gains," IEEE Trans. Commun., vol. 51, no. 3, pp. 416-427, Mar. 2003. 